\title{
PIONERAS DE LA LITERATURA EN GUATEMALA: MUJERES INTELECTUALES, MERCADOS GLOBALES Y CONSUMO FEMENINO
}

\author{
Patricia ArRoyo Calderón \\ University of California, Los Angeles
}

\begin{abstract}
En este artículo se explora la obra de un grupo de escritoras, maestras y periodistas activas en la Ciudad de Guatemala durante las décadas de 1880 y 1890, que fueron pioneras de las letras femeninas centroamericanas y que lograron conectarse con algunas de las redes intelectuales continentales y transatlánticas más importantes de su época. Este grupo estuvo compuesto por figuras como Vicenta Laparra de la Cerda, Jesús Laparra, Rafaela del Águila, Adelaida J. Chéves, Pilar Larrave de Castellanos, Carmen P. de Silva y Sara María García Salas de Moreno. El texto analiza la posición de centralidad que estas mujeres lograron adquirir en el marco de la nueva institucionalidad republicana instaurada por la Reforma Liberal de 1871, para pasar a continuación a valorar la incidencia de estas mujeres intelectuales en algunos de los debates públicos más importantes de su época. En concreto, este artículo presta especial atención a una serie de textos en los que estas autoras critican las nefastas consecuencias que la abundancia de bienes de consumo importados estaba causando en unas sociedades del istmo que recién se estaban abriendo a los mercados globales, y sostiene que estos escritos deben ser leídos como una respuesta creativa - específicamente articulada por mujeres intelectuales - ante las tensiones y las dislocaciones que los procesos de modernización económica estaban generando en la región.
\end{abstract}

PALABRAS CLAVE: Guatemala, redes intelectuales, mujeres escritoras, economía, mercados globales, Vicenta Laparra de la Cerda, Adelaida Chéves, Pilar Larrave de Castellanos, Rafaela del Águila.

Pioneers of Guatemalan Literature: Women Intellectuals, Global Markets, and Female Consumption

This article explores the textual production of a group of women writers, teachers, and journalists -formed by Vicenta Laparra de la Cerda, Jesús Laparra, Rafaela del Águila, Adelaida J. Chéves, Pilar Larrave de Castellanos, Carmen P. de Silva, and Sara María García Salas de Moreno- who were active in Guatemala City during the decades of 1880 and 1890. This group of women were authentic pioneers of women's literature in Central America and were also able to connect with some of the most important hemispheric and transatlantic intellectual networks of their time. This paper analyzes the central position that these women writers occupied within the new educational institutions created after the triumph of the Liberal Reform in 1871, in order to evaluate their impact on some of the most important public debates in late-nineteenth-century Guatemala. In particular, it explores a series of texts where these authors criticized the disastrous consequences caused by the overabundance of imported goods in Central American societies that were quickly opening to global markets. I am interested in arguing that these economic texts

Arroyo Calderón, Patricia (2021), "Pioneras de la literatura en Guatemala: mujeres intelectuales, mercados globales y consumo femenino", Lectora, 27: 47-70. ISSN: 1136-5781 D.O.I.: 10.1344/Lectora2021.27.2, parroyo@ucla.edu

Recepció: 15 de desembre de 2020 - Acceptació: 18 de maig de 2021 
were a creative response, specifically articulated by women intellectuals, vis-à-vis the tensions and dislocations prompted by regional modernization processes.

KEY WORDS: Guatemala, intellectual networks, women writers, economy, global markets, Vicenta Laparra de la Cerda, Adelaida Chéves, Pilar Larrave de Castellanos, Rafaela del Águila.

El 29 de enero de 1905 fallecía en Ciudad de Guatemala, de un paro cardíaco, una conocida escritora de setenta y tres años. Se trataba de la autora Vicenta Laparra de la Cerda: con su desaparición se cerraba un ciclo de extraordinario florecimiento de las letras femeninas en Guatemala. En efecto, entre las décadas de 1880 y 1900, se conformó en esta capital centroamericana una tupida red de maestras, periodistas y escritoras, que incluyó a las hermanas Vicenta y Jesús Laparra, Rafaela del Águila, Adelaida Chéves, Pilar Larrave de Castellanos, Carmen P. de Silva y Sara María García Salas de Moreno. En conjunto, fueron capaces de publicar los dos primeros semanarios del istmo íntegramente redactados por mujeres y destinados a un público lector fundamentalmente femenino, estrenar varias piezas en verso en el Teatro Nacional y publicar al menos tres novelas, así como decenas de poemas, cuentos cortos, ensayos breves y una variedad de materiales pedagógicos destinados a la niñez y la juventud centroamericanas.

Varias de las mujeres pertenecientes a este núcleo intelectual habían pasado buena parte de sus vidas fuera de los confines de la república guatemalteca durante el período de predominio conservador, como resultado de sus vínculos familiares y matrimoniales con connotados políticos y militares liberales. Otras tantas participaron activamente en conferencias regionales e internacionales, incorporándose de este modo a las principales redes literarias e intelectuales centroamericanas y transatlánticas de su época. Algunas de ellas publicaron sus obras en la floreciente industria editorial norteamericana dedicada a la impresión y difusión de textos en español desde la ciudad de Nueva York. Más adelante proporcionaré algunos detalles sobre las experiencias itinerantes de Vicenta y Jesús Laparra por México y la región centroamericana, así como acerca de su participación en congresos y conferencias centroamericanas y transatlánticas. Con respecto a la publicación de obras en empresas editoriales con base en los Estados Unidos, resaltan los contactos que logra Adelaida Chéves para que su manual de economía titulado Llave de oro. Compendio de economía doméstica, para uso de las jóvenes centro-americanas fuera publicado por la Revista Ilustrada de Nueva York en el año 1887.

En este sentido, las mujeres de esta red compartieron experiencias de exilio, desplazamiento, viajes y contactos transnacionales similares a las que marcaron las vidas de la mayor parte de los intelectuales y literatos centroamericanos más cosmopolitas de su época. Asimismo, las mujeres de esta red ocuparon espacios centrales en la vida cultural y en los nuevos espacios educativos abiertos en Guatemala

48 
tras el triunfo de la Reforma Liberal. ${ }^{1}$ La producción textual de estas mujeres fue variada y abundante, y es sin duda parangonable en cantidad — si no también en calidad - a la producida por otras redes de mujeres escritoras, periodistas y pedagogas que operaron en distintas áreas del continente y que sí han recibido la atención de la crítica cultural y literaria latinoamericanista. ${ }^{2}$ El grueso de su obra literaria y ensayística aborda de forma directa algunas de las cuestiones más candentes que se debatieron en el istmo centroamericano durante las décadas finales del siglo XIX, tales como el impacto social de las transformaciones en la estructura de la propiedad y la producción agrarias, el papel que debían desempeñar determinados grupos sociales - como las mujeres, los indígenas o las clases populares- en los

\footnotetext{
${ }^{1}$ Vicenta Laparra de la Cerda regentó una tertulia literaria en su domicilio, a la cual solían asistir connotados personajes de la esfera política y del mundo literario de Guatemala, tales como los escritores costumbristas José Milla y Agustín Mencos Franco, el antropólogo, historiador y hombre de estado Antonio Batres Jáuregui, o el médico, político, historiador y novelista Ramón Salazar (Diccionario, 2004: 874). Asimismo, como se detallará más adelante, varias de las miembros de esta red ocuparon puestos de dirección en las principales revistas pedagógicas de la época.

${ }^{2}$ En los últimos años, el campo de estudio que se centra en la producción textual de las escritoras decimonónicas latinoamericanas ha crecido exponencialmente. Sin embargo, las escritoras e intelectuales centroamericanas del siglo XIX siguen siendo estudiadas de forma muy marginal y a cuentagotas, en trabajos que raramente tratan de insertarlas en dinámicas culturales más amplias a escala continental y/o transatlántica. De entre los estudios disponibles, destaca la inclusión de un reducido número de autoras decimonónicas - como la costarricense Rafaela Contreras de Darío o la hondureña Lucila Gamero de Medina - en las antologías de cuentistas centroamericanas compiladas por Willy Muñoz $(2006,2009)$ y en el Diccionario bibliográfico de narradoras centroamericanas con obra publicada entre 1890 y 2010, de Consuelo Meza Márquez (2011). Asimismo, Helen Umaña (1990) y Janet Gold (1998) incluían en sus precursores trabajos sobre narradoras hondureñas y centroamericanas (respectivamente), estudios sobre la figura y la obra a caballo entre los siglos XIX y XX - de Lucila Gamero. Para el caso de Guatemala, existen estudios sobre pioneras de las letras como la Madre María Teresa de Aycinena (Albizúrez Gil, 2012), Josefa García Granados (Establier Pérez, 2015; Toledo, 2012; Villacorta, 1971), Dolores, más conocida como "Lola", Montenegro (Herrera Peña, 2012 y 2015), y Vicenta Laparra de la Cerda (véase la siguiente nota a pie de página). En términos generales, las aproximaciones desde los estudios literarios a la producción de las mujeres decimonónicas en Centroamérica se caracterizan por su escasez, por su fragmentariedad, y por el estudio individualizado de escritoras concretas. Por otro lado, varias historiadoras se han interesado también por la producción textual de varios círculos de mujeres decimonónicas activos en la región durante las últimas décadas del siglo XIX, abordándolas en este caso desde la metodología de redes intelectuales e interesándose por ellas fundamentalmente en su calidad de agentes de reformas educativas y/o políticas. Entre estos trabajos, pueden destacarse el acercamiento a los círculos de maestras salvadoreñas analizados por Vásquez Monzón (2014), a los de las primeras feministas nicaragüenses elaborados por González-Rivera (2011), o el muy reciente trabajo de Patricia Harms (2020) sobre la red de mujeres que se analiza en este artículo.
} 
procesos de modernización económica de la región, o las consecuencias que la apertura de los mercados locales a la importación masiva de objetos de lujo procedentes de las metrópolis europeas y norteamericanas estaba teniendo en las subjetividades y los patrones de consumo de las mujeres centroamericanas.

A pesar de la variedad de su obra y de los espacios centrales que ocuparon en la conformación de la esfera pública guatemalteca durante las décadas de $1880 \mathrm{y}$ 1890 , los trabajos dedicados a esta red de mujeres intelectuales son aún escasos y, sin duda, parciales. Si bien algunos aspectos de su obra —en especial las dos publicaciones periódicas que este grupo de autoras editó durante la década de 1880, así como varias de las obras de teatro y de las novelas sentimentales escritas por $\mathrm{Vi}$ centa Laparra de la Cerda - han recibido la atención de críticas como Arroyo Calderón (2012), Monzón (2011), Rodas (2006) o, más recientemente, Harms (2020), ${ }^{3}$ estas aproximaciones resultan todavía episódicas y fragmentarias, y dejan aún una multitud de aspectos por explorar. Tratar de ofrecer una explicación exhaustiva acerca de las razones de esta ausencia de atención crítica excede, con mucho, tanto las pretensiones como las posibilidades de este artículo. No obstante, me gustaría mencionar brevemente algunos factores que podrían explicar la desatención y el virtual olvido al que han sido sometidas las autoras de esta red. En primer lugar, podemos destacar la situación de híper-visibilidad de la que gozan ciertos autores centroamericanos del período de entre siglos —especialmente el nicaragüense $\mathrm{Ru}$ bén Darío y el guatemalteco Enrique Gómez Carrillo- en los estudios literarios

\footnotetext{
${ }^{3}$ Patricia Arroyo Calderón ha explorado los textos sentimentales producidos por esta red, vinculándolos con los debates acerca de la incorporación de las mujeres, los indígenas y las clases populares a la ciudadanía que se llevaron a cabo en Guatemala en las últimas décadas del siglo XIX. Por su parte, Ana María Rodas y Ana Silvia Monzón han analizado la figura de Vicenta Laparra de la Cerda y las publicaciones periódicas La Voz de la Mujer y El Ideal, respectivamente, como pioneras de un incipiente movimiento feminista en Guatemala. Por su parte, Patricia Harms es quien mejor ha explorado el impacto que tuvieron las mujeres de esta red y sus escritos en la conformación de nuevos núcleos de mujeres intelectuales en Guatemala en las décadas de 1910 y 1920. Finalmente, existen un par de tesis de licenciatura —Cruz (2000) y Vásquez Monroy (2012) - dedicadas al estudio del semanario El Ideal y de la obra de teatro de Vicenta Laparra de la Cerda El ángel caído. A pesar de estas importantes aproximaciones, aún quedan muchos aspectos por esclarecer de la obra de estas autoras; entre ellos, pueden mencionarse los siguientes: la reconstrucción de su corpus textual; la exploración de los escritos que abordan específicamente debates relacionados con la transformación de las economías del istmo a fines del siglo XIX; la indagación en los vínculos que estas mujeres mantuvieron con otras redes de mujeres coetáneas articuladas en la región - por ejemplo, con el núcleo de maestras salvadoreñas ya estudiado por Vásquez Monzón (2014) o con la hondureña Lucila Gamero-; o sus posibles vínculos con otras redes de mujeres escritoras del mundo hispano - en especial españolas, mexicanas y peruanascuyos nombres aparecen frecuentemente citados en sus textos, y cuyas obras resuenan fuertemente en algunos de los textos producidos por las guatemaltecas.
}

50 Lectora, 27 (2021): 47-70. ISSN: 1136-5781 D.O.I.: 10.1344/Lectora2021.27.2 
latinoamericanos, que va paradójicamente acompañada de un fenómeno simultáneo de infravisibilización de las complejidades del campo cultural y literario decimonónicos en el istmo. En este sentido, el hecho de que los escritos de estas mujeres se alejen mucho de las nuevas sensibilidades estéticas y de los derroteros temáticos impulsados por el modernismo literario no contribuye, probablemente, a su inserción en el campo de estudio de las literaturas latinoamericanas finiseculares. Por otro lado, y a pesar de que el campo de los estudios literarios y culturales centroamericanos es una disciplina en franco proceso de expansión, la forma "presentista” en la que se está configurando este campo - en especial en lo que respecta a la academia norteamericana - tiende a privilegiar de manera notoria el estudio de las manifestaciones literarias y culturales contemporáneas, particularmente lo que ha venido a llamarse las "literaturas centroamericanas de posguerra" (Ortiz Wallner, 2012). Finalmente, un detalle no menor se relaciona con la fragmentación, las ausencias y las dificultades de acceso a los archivos centroamericanos. Si bien todos los investigadores e investigadoras que exploran la textualidad de las mujeres decimonónicas en el ámbito hispano comparten estas mismas dificultades en torno a la construcción del canon y al archivo, la situación de precariedad a la que se enfrentan muchos repositorios documentales en Centroamérica presenta retos específicos relacionados tanto con los procesos de destrucción y abandono causados por conflictos civiles que en algunos casos - el de Guatemala, por ejemplose extendieron durante décadas, como por unos contextos de posguerra marcados por la falta de inversión en instituciones educativas y culturales, así como por una altísima volatilidad política, económica y social.

Desde ese estado de la cuestión sobre el estudio de las literaturas y prácticas culturales de las mujeres decimonónicas centroamericanas, en las páginas que siguen me propongo ofrecer una breve reconstrucción de la red de mujeres escritoras que estuvo activa en la capital de Guatemala durante las dos últimas décadas del siglo XIX, así como ofrecer una vista panorámica del conjunto de obras que publicaron. Por último, me centraré en el análisis de una serie de textos — todavía prácticamente sin abordar por parte de la crítica - a partir de los cuales estas autoras intervinieron en los debates económicos de su época. En concreto, analizaré una serie de manuales y artículos de prensa en los que las autoras señalaban algunas de las dislocaciones que, a su juicio, se habían producido en el istmo a raíz de la acelerada inserción de la región en los mercados globales. En dichos textos, este núcleo de autoras delineaba el rol que las mujeres centroamericanas debían desempeñar en los procesos locales de acumulación de capital, amenazados - a sus ojos- por la emergencia de nuevos patrones de consumo marcados por la irracionalidad y el desenfreno. 


\section{Una red de mujeres en Ciudad de Guatemala}

La tupida red de escritoras, periodistas y maestras que nos ocupa estuvo activa en la Ciudad de Guatemala entre las décadas de 1880 y 1900. Todas sus miembros nacieron entre 1830 y 1860, excepto Jesús Laparra, conocida como la "poetisa mística”, quien nace en 1820 y fallece tempranamente, en 1887. Asimismo, todas ellas estuvieron vinculadas a los proyectos culturales y educativos impulsados desde distintas plataformas y espacios por las dos figuras centrales que articulan la red: Vicenta Laparra de la Cerda y Rafaela del Águila.

Vicenta Laparra de la Cerda fue sin duda la más prolífica de las miembros de la red. Nacida en Quetzaltenango, una ciudad con una fuerte presencia de población indígena maya k'iche', su vida comparte rasgos con la de muchos otros intelectuales de la época. Pasó casi toda su infancia y su juventud en el exilio, dado que tanto su padre, primero, como su esposo, después, compartieron inclinaciones liberales en una época en la que la vida política de la República de Guatemala se encontraba bajo el férreo control del dictador conservador Rafael Carrera (18441865). La vida itinerante de Vicenta Laparra durante ese período la condujo desde Comitán de las Flores, en Chiapas, hasta Santa José de Costa Rica, donde se desempeñó como directora del Colegio Nacional de Señoritas entre 1856 y 1860. Posteriormente, esta autora reaparecerá en Santa Ana, la capital cafetalera de la región occidental de El Salvador, donde ejerció como directora de la Escuela Nacional de Niñas hasta 1866. En ese mismo año, ya fallecido el presidente conservador vitalicio Rafael Carrera, Vicenta Laparra regresa con su familia y se instala en la Ciudad de Guatemala, donde abrirá un pequeño colegio para niñas dentro de su propia casa. En ese momento, el rastro de esta autora se pierde durante varios años, probablemente porque tras el nacimiento de su octavo hijo queda completamente paralizada durante casi diez años (un trastorno que le valdría más adelante el sobrenombre de "la poetisa cautiva"). Vicenta Laparra va a reaparecer, no obstante, con gran fuerza en el centro de la vida cultural guatemalteca en la década de 1880, cuando varias fuentes la sitúan como la anfitriona de la tertulia literaria más concurrida de la ciudad, donde se reunían importantes personalidades culturales y políticas afines a los gobiernos del liberal Manuel Lisandro Barillas - un presidente de origen quetzalteco, al igual que la familia Laparra. En un período de veinte años, Laparra de la Cerda publicó y llevó a la escena con gran éxito de crítica y público al menos cinco obras de teatro que fueron estrenadas en el Teatro $\mathrm{Na}$ cional, renombrado como Teatro Colón en 1892. ${ }^{4}$ También fue la autora de varias novelas sentimentales, de las cuales se preservan dos: La calumnia, de 1894,

\footnotetext{
${ }^{4}$ Se trata de El ángel caído (1886), La hija de un libertino (1886), Los lazos del crimen (1888), La hija maldita (1895) y Tempestades del alma (1896). De todas ellas, la única que no se conserva - hasta donde llega mi conocimiento- es La hija de un libertino.
}

52

Lectora, 27 (2021): 47-70. ISSN: 1136-5781 D.O.I.: 10.1344/Lectora2021.27.2 
y Hortensia, que fue publicada por entregas en la revista de la Escuela Normal Central de Señoritas en el año $1896 .^{5}$ En el apogeo de su fama como literata, Vicenta Laparra fue designada como delegada de Guatemala en diferentes eventos internacionales de carácter cultural y pedagógico, entre ellos el Congreso Literario Hispano-Americano celebrado en Madrid con ocasión del IV Centenario, la Exposición Centro-Americana celebrada en 1893 y el Primer Congreso Pedagógico Centro-Americano, celebrado en Ciudad de Guatemala en ese mismo año, donde Laparra presentó una ponencia que trataba de responder a la pregunta: “¿Cuál será el medio más eficaz de civilizar a la raza indígena, en el sentido de inculcarle ideas de progreso y hábitos de pueblos cultos?" (Primer Congreso Pedagógico Centroamericano, 1894: 372-379).

De su vasta obra, el único aspecto que ha sido concienzudamente analizado es su labor como impulsora de dos importantes publicaciones periódicas. Se trata de los semanarios La Voz de la Mujer (1885) y El Ideal (1887-1888), que fueron las primeras publicaciones periódicas en la región centroamericana íntegramente escritas por mujeres y destinadas fundamentalmente a un público femenino. La Voz de la Mujer fue una empresa sin duda modesta que involucró únicamente a las dos hermanas Laparra, Vicenta y Jesús. Se anunciaba como "un periódico [que] saldrá a luz todos los sábados [...] escrito por señoras guatemaltecas" y aspiraba a ser una publicación donde no se hablara ni de la cosa pública - por considerar las redactoras que "la política es campo demasiado estéril para las señoras" (Barrios, 2013: 35) - ni de religión, un asunto sin duda aún espinoso en los meses inmediatamente posteriores al fin de la presidencia de Justo Rufino Barrios, el mandatario liberal que completó uno de los procesos de secularización más acelerados y radicales de todo el continente. Lo que las hermanas Laparra prometían a sus lectores era ocuparse de "aquellos puntos que pertenezcan exclusivamente a la mujer, según el interesante papel que ella tiene que representar en la escena social" (Barrios, 2013: 36), además de publicar pequeñas novelas — lo cual nunca llegó a ocurriry composiciones poéticas. La vida de La Voz de la Mujer fue corta, ya que Vicenta y Jesús Laparra apenas lograron publicar cinco números; es posible que, a falta de un cuerpo consolidado de lectores, las hermanas no fueran capaces de enfrentarse a los gastos de impresión - algo que parece probable, ya que para editar el último número se vieron obligadas a cambiar de imprenta. ${ }^{6}$ Fuera como fuese, y a pesar

\footnotetext{
${ }^{5}$ Ambas novelas han sido recientemente reeditadas por editoriales locales, la editorial Artemis Edinter y la Tipografía Nacional, gracias al impulso de la Asociación Vicenta Laparra de la Cerda, una agrupación de índole cultural fundada en 2001 por descendientes directos de la autora, dedicada a la divulgación de la obra de esta escritora, así como al fomento de su estudio.

${ }^{6}$ En su libro Ladina Social Activism in Guatemala (2020), Patricia Harms sugiere que La Voz de la Mujer fue víctima de la censura del presidente Manuel Lisandro Barillas. Sin descartar por completo dicha interpretación, considero más probable que La Voz de la Mujer pasara a mejor
} 
de la corta vida de La Voz de la Mujer, resulta evidente que las voces de las Laparra fueron escuchadas por otras guatemaltecas ávidas de tener una plataforma para hacer oír las suyas propias.

De este modo, apenas un año después de la desaparición del primer semanario, Vicenta Laparra regresa a la esfera pública centroamericana y esta vez viene con un nuevo proyecto que implicaba un trabajo colectivo, así como integrar espacios y voces transnacionales. Como anunciaba el primer número de El Ideal, puesto a disposición del público el 10 de diciembre de 1887, este periódico semanal aspiraba a convertirse en el "Órgano de los Intereses de la Mujer" y estaba "redactado solamente por Señoras” (Barrios, 2013: 87). El Ideal seguía así los pasos de otros periódicos continentales exclusivamente redactados por mujeres, como $\mathrm{La}$ mujer, fundado en 1878 y dirigido por la colombiana Soledad Acosta de Samper o Violetas del Anáhuac, una publicación prácticamente contemporánea de El Ideal, dirigida desde México sucesivamente por Laureana Wright de Kleinhans y Mateana Murguía de Aveleyra. ${ }^{7}$ Sin embargo, la inspiración más directa de El Ideal sería El Álbum de la Mujer, una revista fundada y dirigida desde la Ciudad de México por la española Concepción Gimeno de Flaquer. ${ }^{8}$ En efecto, en un artículo titulado "Situación de El Ideal", Vicenta Laparra menciona explícitamente que la intención del semanario centroamericano había sido la de "alentar a las señoras guatemaltecas a que, siguiendo el ejemplo de la ilustre escritora Doña Concepción Gimeno de Flaquer, expresen sus ideas por medio de la prensa" (Barrios, 2013: 243). La admiración que esta red de intelectuales guatemaltecas sentía por la labor

vida de muerte natural. Las hermanas Laparra, y más tarde Vicenta Laparra y el resto del cuerpo de redactoras de El Ideal, expresaron en múltiples instancias las dificultades financieras a las que se enfrentaban tratando de sacar adelante la empresa de publicar semanarios íntegramente redactados por mujeres, y manifestaron su desmayo ante la falta de apoyo a sus actividades de fomento de la cultura femenina.

${ }^{7}$ Sobre Soledad Acosta de Samper, así como sobre su labor periodística al frente de La Mujer, véanse Alzate (2015) y Acosta Peñaloza, Alzate Cadavid y Licón Villalpando (2014). Sobre la publicación Violetas del Anáhuac, véase Infante Vargas (1996).

${ }^{8}$ Concepción Gimeno de Flaquer fue una periodista y escritora de origen español que se radicó en México en 1883. En ese mismo año funda el semanario ilustrado El Álbum de la Mujer, una publicación que sobrevivirá hasta 1890. El Álbum de la Mujer pretendía ser "un monumento consagrado al bello sexo [...] una recopilación de todo lo más instructivo, moral y ameno", dedicado a "las bellas mexicanas" entre las cuales, según Gimeno de Flaquer "no hay mujeres frívolas" (“Saludo", 1883: 2). La vocación de esta publicación era la de instruir deleitando y su carácter era misceláneo: en él se publicaban biografías de mujeres notables, poemas, novelas por entregas, reproducciones de artículos periodísticos de connotados autores españoles y europeos, y noticias de interés cultural o social. Sobre Concepción Gimeno de Flaquer y El Álbum de la Mujer, véanse Garrigan (2016) y Hernández Carballido (2012).

54

Lectora, 27 (2021): 47-70. ISSN: 1136-5781 D.O.I.: 10.1344/Lectora2021.27.2 
periodística de la escritora hispano-mexicana parece haber sido recíproca, ya que la propia Concepción Gimeno de Flaquer seleccionaría al menos un artículo de $E l$ Ideal para ser reproducido en la primera plana de su semanario ilustrado. ${ }^{9}$ Este hecho, junto con la información que proporcionan las propias redactoras de $E l$ Ideal en relación con los intercambios que fueron capaces de establecer con una serie de publicaciones no especificadas de El Salvador, Nicaragua y Colombia, son buenos indicadores que nos permiten afirmar que la labor cultural de esta red de mujeres fue capaz de traspasar las fronteras de Guatemala.

Durante su andadura de veinte números, el semanario El Ideal publicará una plétora de poemas, dedicatorias, ensayos originales sobre temas morales, pedagógicos y sociales, así como la reproducción de un artículo sobre "La mujer estudiosa”, de la ya citada Concepción Gimeno de Flaquer. También publicará relatos por entregas, anuncios sobre actividades benéficas, reclamos comerciales, pequeñas efemérides, anuncios, breves reseñas de publicaciones y repetidos llamamientos a los suscriptores del periódico que, si bien se declaran numerosos, al parecer no eran demasiado aficionados a abonar sus cuotas a tiempo. ${ }^{10}$ Por último, estos semanarios también publicaron una variedad de ensayos sobre la correcta administración del hogar, a los que dedicaré mi atención un poco más adelante.

Las mujeres que acompañarán a Vicenta Laparra en el cuerpo de redacción de El Ideal serán las poetas Isabel M. de Castellanos, Sara María García Salas, y Carmen P. de Silva, cuyo esposo, Felipe Silva, era autor de un diccionario de lenguas mayas y un conocido dramaturgo de piezas de temática indigenista, además de muy convenientemente - el dueño de la imprenta en la que se imprimía El Ideal. Además de las mujeres citadas, se unieron al proyecto dos figuras importantes, las maestras Rafaela del Águila y Adelaida Chéves, cuyo padre financiaba la publicación del semanario gracias a los ingresos obtenidos en la fábrica de hielo y aguas gaseosas que poseía en el departamento de Retalhuleu. Junto con Vicenta Laparra,

\footnotetext{
${ }^{9}$ En concreto, se trató del artículo "La suegra", firmado por Carmen P. de Silva. Este texto fue publicado originalmente en el $\mathrm{n}^{\circ} 3$ de El Ideal (24 de diciembre de 1887) y, cerca de tres meses más tarde, reproducido en El Álbum de la Mujer ( $\mathrm{n}^{\circ}$ 10, año VI, tomo 10, 4 de marzo de 1888).

${ }^{10}$ En el segundo número de la publicación, las redactoras se congratulaban de la excelente acogida que "la buena sociedad de esta capital [...] ha dado a nuestra humilde publicación; pues cosa inusitada, desde el primer número contamos ya con 400 suscriptores" (Barrios, 2013: 110). A la altura del séptimo número, sin embargo, el agradecimiento daba paso a cierta impaciencia cuando las redactoras se veían obligadas a suplicar "a nuestros suscriptores, tanto de esta capital como de los departamentos, se sirvan pagar la suscripción" (154). En el decimoquinto número, un largo artículo salido de la pluma de Vicenta Laparra de la Cerda explicaba la precaria situación financiera de la publicación, la cual había tenido que sortear "mil dificultades, y que si no fuera por la constancia y empeño de nuestro amigo Silva [el impresor], hace mucho tiempo que $E l$ Ideal estuviera sepultado en la tumba del olvido" (244).
} 
Rafaela del Águila y Adelaida Chéves formaron parte de los círculos de pedagogos y pedagogas a los que el régimen de Justo Rufino Barrios encargó la expansión y la laicización del sistema de enseñanza en Guatemala. Las tres mujeres mencionadas habían participado como delegadas en el Congreso Pedagógico de 1881 y formaron parte de la Academia Central de Maestros de Guatemala, creada en ese mismo año. Posteriormente, Rafaela del Águila ocupó diversos puestos importantes, siempre relacionados con el ámbito de la educación de las niñas y con la formación de maestras. Fue directora de la Academia de Maestros durante varios períodos y bajo sus mandatos se crearon las revistas pedagógicas La Academia de Maestros y La Escuela Normal, una publicación que circulaba por todas las repúblicas centroamericanas con el fin de homogeneizar los sistemas educativos de la región. A partir de 1893 — por designación del presidente José María Reina Barrios- del Águila fue nombrada directora de la Escuela Normal Central de Señoritas, un centro especializado en la formación de maestras de educación primaria (Nivón, 2019). Los intereses pedagógicos de Rafaela del Águila recaían, sobre todo, en el campo de la educación moral; como ejemplo, podemos mencionar su cartilla titulada Nociones de educación moral, escritas para los niños, publicada en 1894 y que fue adoptada como libro de texto por todas las escuelas primarias de la república. Desde su posición de dirección, Rafaela del Águila acogió como profesoras del Instituto Belén, nombre con el que era conocida la Escuela Normal Central de Señoritas, a otras miembros de la red con intereses en la creación literaria y en la economía doméstica, como Adelaida Chéves y Pilar Larrave de Castellanos. ${ }^{11}$

Por lo tanto, en este apartado, mi intención ha sido resaltar la densidad de esta red de mujeres intelectuales, la amplitud y la variedad de su producción textual y, finalmente, los espacios centrales que lograron ocupar en el campo de la literatura, de la cultura y de la pedagogía durante las últimas décadas del siglo XIX. En la siguiente sección, me centraré en vincular los escritos de estas mujeres con algunos de los debates más candentes que se dirimieron en los espacios públicos de la república de Guatemala durante las últimas décadas del siglo XIX.

\section{Intervenciones femeninas en los debates económicos}

Sin duda, las escritoras que nos ocupan intervinieron de forma muy activa en los debates en torno a la entonces llamada "cuestión de la mujer". Entre el corpus de textos producido por las miembros de esta red, encontramos poemas, ensayos, ponencias, relatos y fragmentos de novelas dedicados a defender el derecho de las

\footnotetext{
11 Adelaida Chéves publicó, como ya se ha mencionado previamente, el manual de economía doméstica titulado Llave de oro en 1887. Asimismo, en 1890 publicó la novela titulada Clementina, o la víctima de un crimen. Por su parte, Pilar Larrave de Castellanos publicaría en 1895 otro manual, esta vez titulado Economía doméstica, para uso de las jóvenes centroamericanas.
}

56

Lectora, 27 (2021): 47-70. ISSN: 1136-5781 D.O.I.: 10.1344/Lectora2021.27.2 
mujeres a la educación, a la escritura, y a recibir un salario digno por su trabajo. La concepción que tienen todas estas autoras de "la mujer" es limitada y se corresponde, fundamentalmente, con mujeres ladinas, urbanas y letradas. Esto no quiere decir que, en el conjunto de su obra, no reflexionen - ya sea de forma directa o indirecta- sobre ciertas "diferencias" entre sectores distintos de mujeres. En concreto, en las páginas de La Voz de la Mujer y El Ideal van a prestar especial atención a las diferencias de clase social, especialmente en lo que tiene que ver con las distintas funciones que atribuyen a la educación práctica de las guatemaltecas. De acuerdo a estas autoras, los conocimientos de economía doméstica y la consiguiente capacidad de hacerse cargo de las tareas reproductivas de forma eficiente deberían ser imprescindibles para las jóvenes de clase acomodada que, si bien en circunstancias normales podían limitarse a "mandar sus criados, cuidar del orden, del aseo de su casa, tocar el piano, vestirse con elegancia, y esperar tranquilamente el término de su viaje por el mundo", en el caso de carecer de facultades prácticas $\mathrm{y}$ verse afectadas por un revés de la fortuna, quedarían condenadas a la pobreza más abyecta. Por otra parte, también se reflexiona sobre la necesidad de que las mujeres de la clase proletaria "cultiven su inteligencia y aprendan a trabajar" en planteles especializados, ya que la falta de cualificaciones profesionales condenaba, siempre según las escritoras, a las jóvenes de las clases populares a "hacer más, trabajar, afanarse, fatigarse mucho y medrar poco" (Barrios, 2013: 46). Finalmente, prácticamente ninguno de los escritos producidos por esta red intelectual se ocupa de las mujeres indígenas, al menos de forma directa, con la excepción del ensayo presentado por Vicenta Laparra de la Cerda en el Congreso Pedagógico Centroamericano de 1893. No obstante, en sus textos dedicados al servicio doméstico podemos percibir abundantes estrategias indirectas de racialización de las mujeres dedicadas al trabajo doméstico remunerado que, como bien sabemos por los trabajos de Aura Cumes (2014), en Guatemala ha sido históricamente realizado por mujeres indígenas. ${ }^{12}$ Es decir, una intervención fundamental en La Voz de la Mujer y El Ideal fue pensar la educación como un imperativo que, dependiendo de la clase social, permitiría mayor racionalización de las tareas domésticas o mejores condiciones en la vida laboral.

Por otra parte, también encontramos textos dedicados a discutir las responsabilidades de las mujeres como madres y como esposas o a plantear cuestiones relacionadas con el rol de las mujeres en la vida política y social de las repúblicas

\footnotetext{
${ }^{12}$ Para un análisis en profundidad de las estrategias de racialización presentes en los textos de esta red de mujeres decimonónicas, las formas de desvalorización del trabajo doméstico asalariado realizado por las mujeres racializadas que aparecen en los manuales de economía del periodo, así como sus implicaciones para los imaginarios contemporáneos sobre el trabajo doméstico realizado por mujeres mayas, véase Arroyo Calderón (2020).
} 
centroamericanas. Al mismo tiempo, estas escritoras participaron activamente en otros debates fundamentales del período, como la cuestión de "el problema del indio", con textos importantes como la ponencia que Vicenta Laparra de la Cerda presentó en el Primer Congreso Pedagógico Centroamericano de 1893. En su ponencia, Vicenta Laparra señalaba la urgencia de incorporar a la población indígena de manera efectiva a las actividades que garantizarían, a su juicio, el progreso de Guatemala. En concreto, esta autora defendía la necesidad de "educar" a los indígenas para que, de un estadio de "miseria", "abyección" y "abandono", pudieran convertirse en "útiles", "honestos” y "productivos” trabajadores agrícolas. En el esquema de Laparra, "educar" a los pueblos indígenas significaba alfabetizarlos, castellanizarlos, adoctrinarlos en las creencias católicas y, sobre todo, hacer que "olvidasen su pasado" (1894: 377) para lograr integrarlos en un modelo monolingüe y monocultural de modernidad. En este sentido, los argumentos de Laparra de la Cerda son similares a los de otros intelectuales de su época que, como Antonio Batres Jáuregui, exponían tesis parecidas en obras como Los indios, su historia y su civilización (1893). No obstante, los posicionamientos de Laparra se alejan de los de sus contemporáneos masculinos al menos en dos aspectos importantes. Por un lado, esta autora expresa explícitamente su oposición a las políticas de fomento de la inmigración de agricultores europeos a Guatemala, por considerar que "en nuestro caso, la inmigración sería el golpe de gracia para los pobres aborígenes que ya no encontrarían trabajo [...] y el hijo de la selva huiría, buscando en las tierras montañosas donde aún no ha penetrado la planta del hombre [...] un sitio agreste donde levantar su pobre covacha" (Laparra, 1894: 378). Por otro lado, se inclina por implementar políticas que separen a los niños indígenas de sus padres "siquiera fuese por algún tiempo, mientras los niños olvidaban sus primitivas y pésimas costumbres" (377). Según esta autora, eran las sociedades benéficas y las familias ladinas "de buena voluntad" quienes debían proceder cuanto antes a cortocircuitar las posibilidades de transmisión y sobrevivencia de las culturas mayas de Guatemala.

Asimismo, las mujeres de esta red se ocuparon de fenómenos novedosos, como los procesos de secularización y las profundas transformaciones de las instituciones políticas iniciadas por los regímenes liberales instaurados en todos los países de la región entre 1870 y 1893 . Algunas de estas mujeres, como la propia Vicenta Laparra y su hermana Jesús, fueron muy críticas con unos procesos de secularización que consideraban peligrosos para la estabilidad social y política del istmo, y con sus textos construyeron para las mujeres centroamericanas un espacio central para la vida pública en calidad de repositorios de una serie de virtudes

58 
cristianas que consideraban imprescindibles para la convivencia pacífica en unas repúblicas periódicamente desgarradas por guerras fratricidas. ${ }^{13}$

En las páginas que restan de este artículo, me gustaría sin embargo centrarme en el tipo de textos que responden directamente a las profundas transformaciones estructurales sufridas por el tejido económico y social del istmo a raíz de los acelerados procesos de concentración de tierras impulsados por el gobierno liberal de Justo Rufino Barrios y que culminaron, por un lado, en la desamortización de los bienes eclesiásticos y, por otro, en la disolución de las comunidades indígenas y sus tradicionales sistemas colectivos de tenencia de tierras. Estos procesos de concentración del agro productivo en manos de grandes finqueros instituyeron un nuevo modelo de capitalismo agrario mono-exportador y contribuyeron a la inserción de Guatemala en los circuitos globales de exportación de productos altamente rentables, como el café. En este contexto, las escritoras de la red van a movilizarse para producir una serie de textos destinados a reevaluar la función económica de las mujeres en las sociedades "modernas" - es decir, plenamente insertas en los mercados globales del capitalismo finisecular - desde su posicionalidad específica como administradoras del trabajo doméstico y de las finanzas del hogar. Los textos a los que me estoy refiriendo son una serie de artículos, ensayos y, especialmente, dos manuales de economía doméstica escritos por Adelaida Chéves y Pilar Larrave de Castellanos.

Estos textos se centran en cuestiones pedestres y ciertamente poco literarias, como el zurcido de calcetines, la preservación de la manteca o el correcto ventilado de las habitaciones; no obstante, al mismo tiempo, dejan muy claro que las élites letradas del período se encontraban firmemente convencidas de que la educación económica de las mujeres era un requisito fundamental para que las repúblicas centroamericanas fueran capaces de "civilizarse" y "progresar". De hecho, una de las características más notables del modo en el que las reformas liberales se desarrollaron en el istmo tiene que ver con la urgencia manifestada por las autoridades locales por incorporar a los diferentes sectores de la población en actividades orientadas a la producción capitalista. Por un lado, durante las últimas décadas del

\footnotetext{
${ }^{13}$ Este tema ha sido desarrollado con detalle en Arroyo Calderón (2012). No obstante, quedan sin duda numerosos aspectos por explorar acerca de las diferentes reacciones manifestadas por las mujeres intelectuales del istmo ante estos procesos de secularización, ya que distan mucho de ser homogéneas. Por ejemplo - y como claro contraste al posicionamiento expresado por las hermanas Laparra - otras escritoras de este período, tales como la hondureña Lucila Gamero, escribieron furibundos textos anticlericales en los que atacaban con igual fiereza la lascivia de los curas célibes, la venalidad del sistema de justicia y la corrupción de las autoridades locales. El mejor ejemplo de este tipo de prosa es la novela Blanca Olmedo (1904), donde la heroína epónima - una joven institutriz con amplio conocimiento del ensayo de Michelet, Le prêtre, la femme et la famille (1863) - es acosada sexualmente por un lúbrico sacerdote llamado Benigno Sandino.
} 
siglo XIX, podemos ver un esfuerzo por parte de los gobernantes para diseminar las ideas económicas liberales entre los varones de las clases dominantes, que se plasmó en la importación, traducción y circulación de manuales de economía política que ya eran populares en España, Estados Unidos y Francia, así como en la escritura por parte de autores locales de nuevos textos de economía política adaptados al medio y las condiciones específicas de Centroamérica. ${ }^{14}$ Simultáneamente, las autoridades del istmo pusieron en marcha un conjunto de proyectos encaminados a modernizar también las prácticas económicas de las mujeres, los artesanos urbanos y la población indígena. Todos estos proyectos partían de una premisa similar: en el empeño colectivo por acumular capital y lograr el progreso material de la nación, todos los centroamericanos debían contribuir a la creación de riqueza de acuerdo con su posición social particular y a sus habilidades específicas, unas habilidades específicas que debían ser perfeccionadas a partir de procesos de formación diferenciada asociados a espacios educativos segregados. En el caso concreto de Guatemala, de los centros educativos fundados entre 1870 y 1883, las Escuelas de Artes y Oficios (1875), las Escuelas Nocturnas para Artesanos (1878), la Sociedad de Artesanos (1878) y las Escuelas-Talleres para Niños y Niñas Pobres (1883) estarían orientadas a fomentar la educación práctica de las clases populares y los sectores artesanos urbanos, mientras que la Escuela de Agricultura (1870), la Escuela Especial de Indígenas (1879) y el Instituto Agrícola para Indígenas (1883) aspiraban a incentivar la contribución a la economía nacional de las poblaciones rurales y los varones indígenas, respectivamente (González Orellana, 1970: 302322).

La educación económica de las mujeres va a encontrar también su espacio en instituciones especializadas, como la Escuela de Artes y Oficios Femeniles -que estuvo dirigida por Vicenta Laparra de la Cerda en la década de 1890_, en el progresivo aumento de las horas lectivas dedicadas a la "economía doméstica" en el currículum de las escuelas primarias y de las escuelas normales para señoritas y en los esfuerzos ímprobos iniciados por los gobernantes del istmo para atraer maestras belgas, alemanas, suizas y norteamericanas especializadas en esta disciplina.

\footnotetext{
${ }^{14}$ Entre las lecturas de economía política que influyeron en el pensamiento de los intelectuales centroamericanos se encontraban las obras del escocés Adam Smith, así como las de los franceses Joseph Garnier y Henri Baudrillart. En el caso de Guatemala, uno de los mayores impulsores de esta disciplina fue el liberal Lorenzo Montúfar, quien relata en sus memorias que ya desde el año 1870 - un año antes de que se verificara la Reforma Liberal, que dio al traste con décadas de dominio conservador - intentó, con la colaboración del también liberal Miguel García Granados, que se crease una nueva cátedra de Economía Política en la Universidad de San Carlos (Montúfar, 1898: 84-86). Posteriormente, durante uno de sus períodos de exilio en Costa Rica, Montúfar ocuparía la primera cátedra de Economía Política abierta en la universidad de este país, y publicaría su propio manual, Apuntamientos sobre Economía Política (1887).
}

60

Lectora, 27 (2021): 47-70. ISSN: 1136-5781 D.O.I.: 10.1344/Lectora2021.27.2 
Adicionalmente, y de forma similar a lo que había ocurrido con los manuales de economía política, muy pronto comenzaron a importarse manuales de economía doméstica para jóvenes y niñas que ya eran populares en los Estados Unidos, España, Francia y Bélgica, a los que pronto se unió el corpus de textos de producción local mencionado en el apartado anterior de este trabajo. Entre los manuales importados destacan los del español Carlos Yeves, Economía doméstica y labores (1889) y el manual de la norteamericana Florence Atkinson, Economía e higiene doméstica (1888), traducido al español por Appleton, una editorial con base en los Estados Unidos que, entre otras actividades, se dedicaba a traducir y adaptar para el público hispanohablante de Norteamérica, así como para los lectores latinoamericanos, una serie de textos científicos, pedagógicos y divulgativos ya testados en el mercado anglosajón.

El objetivo principal y común de este tipo de publicaciones consistía en familiarizar al público femenino con una serie de conceptos un tanto arcanos tales como "valor", "riqueza”, "ahorro", "dinero", "capital", "trabajo productivo" o "trabajo improductivo", en una forma pragmática y entretenida. Los contenidos "científicos" que introducía la disciplina de la economía doméstica estaban orientados tanto a completar la formación como a disciplinar las subjetividades y los comportamientos de las jóvenes casaderas, para que éstas pudieran contribuir a la economía familiar "haciendo todo lo posible porque el sagrado producto del trabajo de su esposo no se gast[ase] en superfluidades" (Laparra, 2013b [1888]: 290). Como explicaba Vicenta Laparra en su artículo titulado "La pereza", las mujeres debían "aprender la ciencia del hogar" (288) sin importar su extracción social, ya que, si eran pobres, la diligencia y el aseo convertirían su casa en un paraíso y constituirían su única fortuna, mientras que, si eran ricas, los conocimientos de economía doméstica serían la única barrera que se interpondría entre ellas y el despilfarro de la fortuna de su esposo. Precisamente, eran las muchachas de buena familia quienes necesitaban con urgencia un tipo de educación que les permitiera comprender cabalmente sus deberes económicos en la nueva sociedad:

Muchas veces consiste en la mujer la ruina de la familia. El hombre se casa creyendo que va a coger el cielo con las manos; pero resulta que su tierna compañera no sabe hacer nada, absolutamente nada, que necesita una criada para cada oficio, modista para que le arregle los trajes, costurera para no andar rota, nodriza para que amamante a los chiquillos [...]; ama de llaves porque no entiende esa jerigonza de cuartillos y medios y legumbres, y el diablo y el demonio que no le enseñaron en la casa paterna y que no quiere aprender [...]. ¿Qué resulta de esto? Que para el infeliz marido comienzan los apuros, que su capital no puede hacer frente a tan crecidos gastos, que contrae deudas, que no puede cubrir sus compromisos y que 
viene la quiebra y con ella la desesperación y la deshonra. (Laparra, 2013b [1888]: 289)

Para evitar este tipo de situaciones, la educación de las mujeres debía ser reformada para incluir, además de los contenidos más tradicionales, una serie de nuevas enseñanzas que incluyeran técnicas de escritura, retórica, idiomas extranjeros, geografía, música y economía doméstica. ${ }^{15}$ A la altura de la década de 1880 , ya no bastaba con que las centroamericanas desplegasen sus "angelicales alas" en los espacios íntimos del hogar. Más bien, una feminidad moderna bien entendida implicaba la necesidad de reconocer a las mujeres como agentes económicos activos y como sujetos abiertos a los movimientos e influencias del mundo exterior, imprescindibles tanto para la buena marcha de sus hogares individuales como para el éxito de los procesos de acumulación de capital que garantizarían el progreso económico de la nación. En opinión de Vicenta Laparra de la Cerda, la educación de las jóvenes requería de un giro práctico, ya que:

Encanta una señorita hablando del sol y sus satélites, de la vía láctea y de las nebulosas, de la zona tórrida y de los océanos, de las plantas y los minerales; pero si no sabe cómo se trufa y se prensa un pavo, cómo se talla un vestido y se cabecea una media, le falta mucho que aprender, porque puede casarse y entonces son las congojas; y no porque los hombres quieran que las esposas sean criadas de lujo, sino porque es preciso que cada cual cumpla con las obligaciones de su estado. (Laparra, 2013b [1888]: $290)^{16}$

\footnotetext{
${ }^{15}$ En su artículo "La pereza" (2013b), Vicenta Laparra de la Cerda pone como ejemplo el currículum de la escuela que dirigía Concepción S. de Zirión en Ciudad de Guatemala, donde "no sólo cultivan la inteligencia de las alumnas, adornándolas con las flores de las bellas artes, sino que las aplican a los oficios propios del sexo débil. Las discípulas de Doña Concepción S. de Zirión, hablan idiomas, escriben perfectamente, tocan varios instrumentos, hacen viajes en geografía, aprenden retórica y saben disponer una comida, barrer la casa y remendar la ropa. ¡Esa sí que es buena educación!" (290).

${ }^{16}$ En ese mismo artículo (2013b), Laparra continúa con su reformulación del ideal ético y estético del "ángel del hogar" constatando que: "La mujer instruida es un ángel revestido con las preciosas galas del saber; pero su dedicación no será completa si no sabe qué ingredientes debe llevar el cocido ni cómo se limpia una sartén. La joven es poética cuando sentada junto al piano arranca al sonoro instrumento torrentes de notas melodiosas; pero no lo es menos cuando adornada con su delantal blanco y con las llaves prendidas al cinto, recorre la casa dando órdenes y enseñando a las criadas cómo se hacen las cosas o haciéndolas ella, por más que parezca prosaico visitar la cocina y acercarse al lavadero" (290).
}

62 
En estos textos, podemos ver cómo las autoras guatemaltecas vinculadas a esta red entendían que la sacrosanta misión del gobierno del hogar solo podía desempeñarse con eficacia a partir del continuo despliegue de una serie de virtudes económicas diferenciadas en función del género, entre las cuales se contaban la habilidad de las amas de casa para manejar el tiempo de manera productiva, su aptitud para gestionar de forma eficiente los bienes perecederos y no perecederos de su hogar; su capacidad para controlar las actividades y los cuerpos de sus criados o su talento para transformar un entorno de austeridad material en una fuente de placer estético.

La más importante de las nuevas virtudes femeninas iba a consistir, sin embargo, en la capacidad de la mujer para controlar su propio deseo y, como consecuencia de ello, ser capaz de mantener los gastos domésticos bajo un estricto control. Esta disciplina libidinal adquiría tintes dramáticos a medida que el modelo económico agroexportador característico del período se consolidaba en la región. Por un lado, los fantásticos capitales acumulados por las emergentes élites cafetaleras - gracias al alto precio del café en los mercados internacionales- prometían un futuro esplendoroso para las naciones del istmo, siempre y cuando estos capitales fueran juiciosamente reinvertidos en la expansión y la intensificación de las actividades productivas. No obstante, esta promesa de progreso material ininterrumpido peligraba, según los intelectuales de la época, por la omnipresencia de una serie de vicios económicos extendidos entre la población masculina, vicios que abarcaban desde la excesiva inclinación a invertir en bienes inmuebles de poca rentabilidad, hasta la inmoderada propensión hacia el alcohol y los juegos de azar. No menos grave era la afición de las mujeres por el consumo suntuario, más preocupante si cabe en un momento en el que los objetos de lujo importados de Europa - telas, trajes, accesorios, objetos decorativos, mobiliario, etc.- comenzaban a inundar los mercados locales, hasta el punto de que el escritor José Milla rebautizó Ciudad de Guatemala como "Tendópolis" en uno de sus famosos cuadros de costumbres. ${ }^{17}$ Como expresaba con angustia Pilar Larrave de Castellanos en 1895:

\footnotetext{
17 Se trata del cuadro "Plaza Mayor y tiendas. Cuestión gravísima", compilado en el volumen Libro sin nombre, que salió a la luz en 1870. En este cuadro, Milla manifiesta que "[1]a ciudad aparece atacada por un nuevo género de locura que no he visto hasta ahora clasificada en ninguno de los libros que han escrito los alienistas, y que creo poder llamar tendomanía. Tiempo ha que las cocheras antiguas se volvieron tiendas; las casas reducen las piezas habitables que dan a la calle y abren tiendas, y al paso que vamos, Guatemala pronto cambiará su nombre y se llamará Tendópolis. El ayuntamiento se entiendó por sus cuatro costados; y como la manía es contagiosa, temo que de repente, se entiende el palacio de gobierno y el edificio de la corte, y la catedral y acabemos por no entendernos a fuerza de tiendas. Dentro de poco tiempo todo aquel que no tenga siquiera para una tienda y no venda aunque sea papel quebrado y pita de Santa Ana, será
} 
A medida que el lujo en objetos de mera vista se ha aumentado, se hacen pocos capitales. Anteriormente las personas por otro extremo, sólo atesoraban, y para ellas eran como si no existiesen ciertas bagatelas que hoy ninguno se priva de comprarlas [...]. Hoy en todas partes se trabaja con ahínco desde muy temprano hasta con luz artificial, pero tanto se gana, como se emplea, no sólo en lo necesario para sostenerse, sino hasta en lo inútil; por persona de mal gusto se creería la que no tiene en su tocador perfumes, cosméticos, motas, cepillos, etc., tanto más caros cuanto menos necesarios. De poco tono pensaría ser la joven que no cuenta por docenas los guantes, los listones y otras cosas por ese estilo [...]. "No me alcanza lo que gano", dicen con harta frecuencia muchas personas. ¿Y cómo les ha de alcanzar para multitud de objetos que no cubren ninguna necesidad? (134)

Como puede observarse, la reacción de este grupo de escritoras frente a los nuevos fenómenos que se estaban generando en el campo del consumo se encuentra en las antípodas de la fascinación que sus contemporáneos modernistas sintieron por los titilantes objetos de lujo exhibidos en luminosos escaparates. Los trabajos clásicos de Ángel Rama (1985) o Julio Ramos (1989) sobre el modernismo ya registraban la estrecha relación que puede encontrarse entre este movimiento literario y las dramáticas transformaciones sufridas por las economías latinoamericanas en el cambio de siglo. Por su parte, más recientemente, Erica Beckman (2013: 42-79) ha explorado la obra de los modernistas Julián del Casal, José Asunción Silva y Rubén Darío a partir de la conspicua incorporación de lo que esta autora denomina "modernista import catalogues" en un buen número de sus textos tempranos. A juicio de Beckman, "modernismo pioneered an aesthetic language of commodity consumption in Export Age Latin America. [...] [T]hese authors developed signifying practices through which imported objects might be desired, cherished, and indeed 'loved"' (45), lo cual nos señala la centralidad de las nuevas prácticas de consumo de bienes importados en la articulación de los imaginarios latinoamericanos de la modernidad.

Lejos de celebrar la capacidad de embrujo de estos objetos, Larrave de Castellanos y las demás mujeres de esta red constataban los perniciosos efectos sociales provocados por la creciente fetichización de las mercancías importadas, incluyendo el estéril agotamiento de las fuerzas psíquicas y físicas de las poblaciones del istmo a causa de un exceso de excitación de la fantasía, así como por el trabajo desmedido y la deshora. Este diagnóstico llevará a este grupo de mujeres a buscar

declarado miembro inútil de la sociedad, cuya base va a ser de hoy en adelante el toma y daca" (22).

64

Lectora, 27 (2021): 47-70. ISSN: 1136-5781 D.O.I.: 10.1344/Lectora2021.27.2 
soluciones pragmáticas capaces de contener la sangría de energías vitales y de capitales que el consumo femenino desbocado desviaba, cada vez más, desde Centroamérica hacia Europa. Algunas de ellas, como Adelaida Chéves, propondrán un modelo de consumo racionalizado y segmentado en función del poder adquisitivo del comprador; otras, como Pilar Larrave, considerarán que ese tipo de aproximaciones estaban condenadas al fracaso a menos que se interviniese directamente en la raíz del problema: la falta de capacidad de los centroamericanos y las centroamericanas para (auto)controlar su propio deseo.

Siguiendo esta lógica, Pilar Larrave de Castellanos incidirá en el rol fundamental que las mujeres educadas debían desempeñar en la represión de estas nuevas instancias irracionales del comportamiento humano. En concreto, en su manual Economía doméstica, para uso de las jóvenes centroamericanas (1895), Pilar Larrave va a designar a las madres y a las maestras como aquellos agentes encargados de predicar el evangelio del ahorro, de disciplinar los afanes imitativos de sus hijas y sus criados, y de impulsar un nuevo paradigma estético transclasista basado en la sobriedad y la pulcritud tanto en lo que respectaba al arreglo personal como a la decoración del interior de los hogares. Adicionalmente, debían ser estas mismas madres y maestras las encargadas de extirpar el aguijón del deseo de las psiques de sus hijas y pupilas desde su más tierna infancia. Los mejores antídotos para las fantasías de consumo consistían, para esta autora, en la enseñanza de materias que robustecieran el tejido moral de la subjetividad de las centroamericanas, así como la dedicación continuada de las niñas y las jóvenes a las actividades productivas en el seno del hogar. Las escuelas para párvulos, siempre siguiendo los argumentos de Larrave de Castellanos, debían convertirse en espacios donde los niños y las niñas aprendieran "a guardar y a cercenar sus pequeños gustos, por livianos que sean” (1895: 133), mientras que la dedicación sistemática a los quehaceres domésticos, la restricción de las horas de ocio, y la vigilancia permanente durante los paseos, las visitas o los convites sociales eran medidas orientadas a garantizar que no se despertase "el afán de gozar" (102) entre las más jóvenes.

\section{Conclusiones}

En este artículo he presentado la obra de un grupo de escritoras, maestras y periodistas activas en la Ciudad de Guatemala durante las décadas de 1880 y 1890 . Este grupo estuvo encabezado por la figura central de Vicenta Laparra de la Cerda, quien fue acompañada en sus proyectos literarios e intelectuales por un núcleo de mujeres menos conocidas, como su hermana Jesús Laparra, Rafaela del Águila, Adelaida J. Chéves, Pilar Larrave de Castellanos, Carmen P. de Silva y Sara María García Salas de Moreno. A lo largo del texto, he argumentado la importancia de estudiar la trayectoria vital y la producción textual de este círculo de mujeres que no solamente fueron verdaderas pioneras de las letras femeninas centro- 
americanas, sino que fueron también capaces de conectarse con algunas de las redes intelectuales transcontinentales y transatlánticas más importantes de su época. Asimismo, he mostrado la posición de centralidad que lograron adquirir en el marco de la nueva institucionalidad republicana instaurada por la Reforma Liberal de 1871, así como la vinculación de su amplia producción textual con los debates públicos más importantes de su época, incluyendo los debates sobre "la cuestión de la mujer" y sobre "el problema del indio".

Finalmente, he resaltado uno de los aspectos de la obra de estas mujeres que menor atención ha recibido hasta el momento: la vinculación de buena parte de sus textos con las profundas transformaciones que la apertura de las economías centroamericanas a los mercados globales estaba provocando en la región. En concreto, he analizado una serie de escritos orientados a alertar de lo que estas autoras percibían como las consecuencias nefastas que la abundancia de bienes de consumo importados estaba causando en las subjetividades y en los cuerpos de los centroamericanos, así como en la capacidad de acumular capital - y, por tanto, de crecer- de las naciones del istmo. Frente a las lecturas críticas que interpretan este tipo de escritos en contra del consumo y el lujo excesivos como respuestas estrictamente tradicionalistas o conservadoras frente a las nuevas realidades económicas del cambio de siglo, he defendido que, por el contrario, debemos leer estos textos más bien como una respuesta creativa - específicamente articulada por mujeres intelectuales - ante las tensiones y las dislocaciones que los procesos de modernización económica estaban generando en la región.

\section{REFERENCIAS BIBLIOGRÁFICAS}

Acosta Peñaloza, Elisa; Carolina Alzate Cadavid y Azuvia Licón Villalpando (eds.) (2014), La Mujer (1878-1881), de Soledad Acosta de Samper. (Periodismo, historia, literatura), Bogotá, Instituto Caro y Cuervo.

Albizúrez Gil, Mónica (2012), “Porque la fe está muy muerta en el mundo’: escritura y llagas de la Madre María Teresa de Aycinena de la Santísima Trinidad Aycinena Piñol (1784-1841) en la proclamación de otra independencia", Mujeres en el Bicentenario. Aportes femeninos en la creación de la República de Guatemala, Julia Guillermina Herrera Peña (ed.), Guatemala, UNESCO: 37-64.

Alzate, Carolina (2015), Soledad Acosta de Samper y el discurso letrado de género, 1853-1881, Madrid, Iberoamericana-Vervuert.

Arroyo Calderón, Patricia (2012), "La articulación de un imaginario femenino para la modernidad centroamericana (1880-1898)", Mujeres en el Bicentenario.

66

Lectora, 27 (2021): 47-70. ISSN: 1136-5781 D.O.I.: 10.1344/Lectora2021.27.2 
Aportes femeninos en la creación de la República de Guatemala, Julia Guillermina Herrera Peña (ed.), Guatemala, UNESCO: 87-119.

-(2020), "Racismo y desvalorización del trabajo de las mujeres indígenas: desde la economía doméstica hasta el caso de Sepur Zarco", EntreDiversidades: Revista de Ciencias Sociales y Humanidades, 7 (2): 94-126.

Batres Jáuregui, Antonio (1893), Los indios, su historia y su civilización, Guatemala, Tipografía La Unión.

Barrios, Amable (ed.) (2013), La Voz de la Mujer y el Ideal. Periódicos precursores de la prensa femenina en Centroamérica, Guatemala, Tipografía Nacional.

Beckman, Erica (2013), Capital Fictions: The Literature of Latin America's Export Age, Minneapolis, University of Minnesota Press.

Chéves, Adelaida J. (1887), Llave de oro. Compendio de economía doméstica, para uso de las jóvenes centro-americanas, Nueva York, La Revista Ilustrada de Nueva York.

-(1890), Clementina, o la víctima de un crimen, Guatemala, Tipografía La Unión.

Cruz, Hugo (2000), Mujer y modernidad en el discurso del semanario feminista El Ideal, Guatemala (1887-1888), Tesis de licenciatura, Guatemala, Universidad Rafael Landívar.

Cumes Simón, Aura Estela (2014), La “india" como "sirvienta". Servidumbre doméstica, colonialismo y patriarcado en Guatemala, Tesis doctoral, México, CIESAS, 20/10/2014. <http://repositorio.ciesas.edu.mx/bitstream/handle/ 123456789/283/D259.pdf? sequence $=1$ \&isAllowed $=\mathrm{y}>$

Del Águila, Rafaela (1894), Nociones de Moral, escritas para los niños, Guatemala, Tipografía Nacional.

Diccionario Histórico Biográfico de Guatemala (2004), Guatemala, Fundación para la Cultura y el Desarrollo \& Asociación de Amigos del País.

Establier Pérez, Helena (2015), "La construcción del sujeto femenino en las poesías líricas de María Josefa García Granados: una pionera del romanticismo entre dos mundos", Acta Literaria, 51: 67-85.

Gamero de Medina, Lucila (1990), Blanca Olmedo, Tegucigalpa, Editorial Guaymuras. [1904]

Garrigan, Shelley (2016), "El 'pensamiento viril': diálogos entre la ciencia y el género en El Álbum de la Mujer", Cuadernos de Literatura, 20 (39): 131-147.

Gimeno de Flaquer, Concepción (1991), "Saludo”, El Álbum de la Mujer, México, Instituto Nacional de Antropología e Historia: 2. [1883]

Gold, Janet (1998), Volver a imaginarlas. Retratos de escritoras centroamericanas, Tegucigalpa, Editorial Guaymuras. 
González Orellana, Carlos (1970), Historia de la educación en Guatemala, Guatemala, Imprenta de Pineda Ibarra.

González-Rivera, Victoria (2011), Before the Revolution: Women's Rights and Right-Wing Politics in Nicaragua, 1821-1979, University Park, The Pennsylvania State UP.

Harms, Patricia (2020), Ladina Social Activism in Guatemala, Albuquerque, University of New Mexico Press.

Hernández Carballido, Elvira (2012), "Periódicos pioneros fundados por mujeres: Las Hijas del Anáhuac, El Álbum de la Mujer, El Correo de las Señoras y Violetas del Anáhuac (1873-1889)", Derecho a comunicar, 6: 1-20.

Herrera Peña, Guillermina (2012), "El poeta como consagración de lo humano: la utopía de Lola Montenegro”, Mujeres en el Bicentenario. Aportes femeninos en la creación de la República de Guatemala, Julia Guillermina Herrera Peña (ed.), Guatemala, UNESCO: 119-142.

Infante Vargas, Lucrecia (1996), "Las mujeres y el amor en Violetas del Anáhuac. Periódico literario redactado por señoras (1887-1889)", Secuencia, 36: 175-212.

Laparra de la Cerda, Vicenta (1886), El ángel caído: drama original en cuatro actos y en verso, Guatemala, Tipografía de Pedro Arenales.

-(1888), Un ángel en la escena. Guatemala, s/e.

-(1894), "Estudio de doña Vicenta Laparra de la Cerda. ¿Cuál será el medio más eficaz de civilizar á la raza indígena?", Primer Congreso Pedagógico Centroamericano y Primera Exposición Escolar Nacional, Guatemala, Tipografía Nacional: 372-379.

-(1895), Hija maldita: drama en tres actos y un epílogo escrito en verso, Guatemala, Tipografía Nacional.

-(2006), Hortensia, Guatemala, Tipografía Nacional. [1896]

-(2008), Tempestades del alma, Guatemala, Ministerio de Cultura y Deportes. [1896]

-(2013a), "Situación de El Ideal", La Voz de la Mujer y el Ideal. Periódicos precursores de la prensa femenina en Centroamérica, Barrios Amable (ed.), Guatemala, Tipografía Nacional: 243-245. [1888]

-(2013b), "La pereza", La Voz de la Mujer y el Ideal, Periódicos precursores de la prensa femenina en Centroamérica, Barrios Amable (ed.), Guatemala, Tipografía Nacional: 288-291. [1888]

-(2015), La calumnia, Guatemala, Artemis Edinter. [1894]

Larrave de Castellanos, Pilar (1895), Economía doméstica, para uso de las jóvenes centroamericanas, Guatemala, Tipografía Americana.

68

Lectora, 27 (2021): 47-70. ISSN: 1136-5781 D.O.I.: 10.1344/Lectora2021.27.2 
Márquez, Consuelo Meza (2011), Diccionario bibliográfico de narradoras centroamericanas con obra publicada entre 1890 y 2010, Aguascalientes, Universidad Autónoma de Aguascalientes.

Milla, José (1870), "Plaza Mayor y tiendas. Cuestión gravísima”, Libro sin nombre, Guatemala, E. Goubaud y Cía: 20-23.

Montúfar, Lorenzo (1887), Apuntamientos sobre economía política, Guatemala, Tipografía La Unión.

-(1898), Memorias autobiográficas. Primera parte, Guatemala, Tipografía Nacional.

Monzón, Ana Silvia (2011), "Participación social y política", Nosotras las de la historia: mujeres en Guatemala (siglos xix-xx), Guatemala, La Cuerda: 146-206.

Muñoz, Willy (2006), Narradoras costarricenses. Antología de cuentos, San José, EUNED.

-(2009), Huellas ignotas. Antologías de cuentistas centroamericanas (1890-1990), San José, EUNED.

Nivón, Amalia (2019), "Redes intelectuales en la reforma educativa de Guatemala a finales del siglo XIX”, Diálogos. Revista Electrónica de Historia, 20 (1): 56-77.

Ortiz Wallner, Alexandra (2012), El arte de ficcionar: la novela contemporánea en Centroamérica, Madrid \& Fráncfort del Meno, Iberoamericana-Vervuert.

Primer Congreso Pedagógico Centroamericano y Primera Exposición Escolar Nacional Instalados en la Ciudad de Guatemala en Diciembre de 1893 bajo la protección del Señor General Don José María Reyna Barrios, Presidente Constitucional de la República (1894), Guatemala, Tipografía y Encuadernación Nacional.

Rama, Ángel (1985), Las máscaras democráticas del modernismo, Montevideo, Fundación Ángel Rama.

Ramos, Julio (1989), Desencuentros de la modernidad en América Latina: literatura $y$ política en el siglo XIX, México, Fondo de Cultura Económica.

Rodas, Ana María (2006), "Vicenta Laparra de la Cerda: Voz de la mujer en el siglo XIX”, Hortensia, Vicenta Laparra de la Cerda, Guatemala, Tipografía Nacional: 7-11.

Toledo, Aída (2012a), “Acerca de una autora que se resiste al olvido: Josefa García Granados”, Mujeres en el Bicentenario. Aportes femeninos en la creación de la República de Guatemala, Julia Guillermina Herrera Peña (ed.), Guatemala, UNESCO: 65-70.

-(2012b), "Frente al imaginario retrato", Mujeres en el Bicentenario. Aportes femeninos en la creación de la República de Guatemala, Julia Guillermina Herrera Peña (ed.), Guatemala, UNESCO: 71-85. 
Umaña, Helen (1990), Narradoras hondureñas, Tegucigalpa, Editorial Guaymuras.

Vásquez Monroy, María Olimpia (2012), El ángel caído de Vicenta Laparra de la Cerda: drama fundacional del teatro en Guatemala, Tesis de licenciatura, Guatemala, Universidad de San Carlos de Guatemala.

Vásquez Monzón, Olga Carolina (2014), Mujeres en público. El debate sobre la educación femenina entre 1871 y 1889, San Salvador, UCA.

Villacorta, Jorge Luis (1971), María Josefa García Granados: su vida, su obra, su correspondencia, sus papeles, en la leyenda, en el teatro, Guatemala, Editorial de Pineda Ibarra.

Yeves, Carlos (1889), Economía doméstica y labores, Madrid, Librería Vda. de Hernando \& Cía. 